\title{
Social Network Communication: Emojis and EFL learners' Writing Issues
}

\author{
Jeehaan Algaraady \\ Taiz University, Yemen \\ Jihan.amu@gmail.com
}

Mohammad Mahyoob (Corresponding Author)

Science \& Arts College, Alula, Taibah University, Madina, Saudi Arabia

eflu2010@gmail.com

\section{Bio-profiles:}

Dr. Jeehaan Algaraady is currently an Assistant Professor at the English department, Taiz University, Yemen. She received her $\mathrm{PhD}$ in Linguistics (Computational Linguistics) from Aligarh Muslim University. Her research interests include Theoretical Linguistics, Applied Linguistics, Computational Linguistics and e-learning.

Dr. Mohammad Mahyoob is currently working as an Assistant Professor in the Department of Languages \& Translation, College of Science and Arts, Alula, Taibah University, Madina, Saudi Arabia. He holds PhD in Computational Linguistics from Aligarh Muslim University. His research interests include Theoretical and Applied Linguistics, Computational Linguistics and e-learning. ORCID: https://orcid.org/0000-0002-6664-1017Abstract

\begin{abstract}
This study aims to find out the impacts of using emojis by EFL learners on their writing skills and highlight the learners' attitudes towards this new communication phenomenon. It discusses the different uses of emojis in social media apps, investigates the reasons for the rise of using emojis in everyday social interaction, and to which extent the occurrence of this pictographic script can substitute the written language. A qualitative and quantitative analysis has been applied in this investigation where a survey-based questionnaire was distributed among 143 EFL learners in Taibah University in Saudi Arabia. Descriptive statistics and ANOVA (analysis of variables) are used to analyze the obtained data. The results show that the p-value of the study variables is equal to one which is much bigger than alpha and there is no big difference between the variables' estimation in the participants' responses, i.e. the emojis' use
\end{abstract}


in texting affects the use of the language. Moreover, the findings display that the use of emojis and short forms (contractions and acronyms) in text messages form a real threat to the standard and non-standard languages. The outcomes of this study make it clear this new sort of communication may replace mainly languages where social media users found that emojis best represent their feelings and thoughts. This research concluded that the use of emojis has an important role in interpersonal communications, however, standard writing skills would be negatively affected using these newly emerged communication tools. The consequences of these impacts are aptly evidenced in the form of spelling, structural errors, and weakness of expressions in EFL learners' language learning.

\section{Keywords: EFL Learners, Emojis, Social Media Communication, Writing Issues}

\section{Introduction}

Communication is essential to overall social media users, as it is the fastest medium for interaction in media and social networks around the globe. Technology-mediated communication (TMC) is the process that is generated between communication and technological tools evolved and increasingly used through different times (López Get, 2011; Martínez-Lirola, 2012; Burholt \& Windle, 2020). Modernization and globalization played a significant role in language alteration and communication growth. The rapid innovation in the technology and social mediatization evolution played the most important role in the appearance of a new sort of communication tools. As a result, emojis came into being and like emoticons, GIFs and other 'graphicons' (graphical icons, Herring \& Dainas, 2017) are an asset to clarify the meaning of texts (Thompson \& Filik, 2016) or to enhance meaning to it (Derks et al., 2007).

Emojis, also known as ideograms or smileys first introduced as "picture" $(e)+$ "characters" (emoji) in Japanese mobile phones electronic messages, and Web pages around the turn of the $21^{\text {st }}$ century ( $\mathrm{Lu}, 2016$ ). They are added to Unicode 6.0 in 2010, and swept to all languages, nations, and cultures, and changed the social and linguistic landscapes.

Interestingly, they are the only communication medium which can be understood by all users from different languages with different social and cultural background and could enable people to connect and engage more concisely with one another demographic groups (Danesi, 2016). Moreover, emoji as any communicative device does not always display what is going on inside but often reflects the cultural aspect of the users. They instantly send and deliver the users' emotions and say more than words, thus messages need not be typed long. The emoji "Face with Tears of Joy " was even nominated as the "Oxford Dictionaries word of 2015" 
because it best represents the feelings, the ethos, and the concerns of the world in that year ( $\mathrm{Lu}$, 2016). As emojis are widely spread in the social field, all these novel forms of communication have their existence in the educational world and, especially, in higher education (VeytiaBucheli, 2020). As digital communication help learners of English to improve their language skills, conversely, the new sort of social media communication has a negative impact on improving the standard language writing and second language learning because of employing slang language and emojis in social communication among learners. The interest in this study is on highlighting the potential reasons behind the appearance and the usage of emojis by EFL learners in their writings and their effect on language (spelling and writing).

In this spirit, Symbolic Interactionism Theory (SIT) named by Herbert Blumer in 1969 states the theoretical framework of this research study. SIT proposed that symbols meanings are derived from social interaction and it attempted to explain how people attach various meanings to words and symbols to represent their ideas, views, and emotions. On a methodological plane, the authors applied a survey-based questionnaire to collect the data and used ANOVA and descriptive statistics to analyze the obtained data.

The current study attempts to grasp the uses and functions of emojis in social networks among EFL learners, and the consequent effect of the novel communication tool emergence on standard language writing. İt is noted that using different new sources of communications (emojis, emoticons, stickers, slang languages, etc.) by EFL learners negatively affect second language writing skill because the learners moved towards these communication sources and did not follow standard language writing rules and strategies. The rest of the paper is organized as the following, Section 2 provides research questions, section 3 throws light on the existing work and approaches as the Literature Review. Section 3 describes the Proposed Methodology including the research population and sample and data collection instruments, Section 4 deals with the results, depicting the results visualization, section 5 provides the discussion of the study, and finally, it is summed up the study with future scope and recommendation.

\section{Related Work}

\section{Presence of Emojis in Tertiary Education Communication}

This work has focused on the use of emojis in the communication context among university students, including the use of emojis instead of language (Parts of speech) and the expressing feelings more than the language in Social networks. Many pieces of research predating this work. Over recent years, emojis have grown to be a hot topic for study, with the volume of papers increasing gradually from 2015 and peaking at 2017-2019 (Bai et al., 2019). Most 
research utilized empirical studies and concentrating on the different characteristics, attributes, and functions of emojis in communication; the use of emojis in the context of platforms diversity, cultures, and individuals; and the application of emojis in research. There are many contributions with diverse research approaches related to university students' use of emojis in spelling and text messaging (Gómez Camacho, \& Gómez del Castillo, 2015) and the general use of WhatsApp by higher education students (Mahyoob, 2020 \& Gómez del Castillo, 2017). Other research in this area, Dunlap et al. (2016) focused on introducing emoticons as learning tools to improve online teaching and learning among members in a community of practice.

Veytia-Bucheli et al. (2020) identified the frequency of emojis use through WhatsApp amongst Mexican graduate students and highlighted the advantages and disadvantages of their use. Their investigation confirmed the augmented use of emojis in conversations among fellow students. Giraldo et al. (2018) examined grammar and writing with the use of technological tools. Cantamutto and Vela (2019) investigated and analyzed WhatsApp interactions integrating emojis amongst students from Argentina and Spain. Prieto-Terrones and SanzMartin (2019) explored the acceptance of writing through WhatsApp, where it is known and accepted by users. They stressed the simplicity it has for rapid communication.

Rodríguez-Morales and Rodríguez-Salazar (2016) studied the young people's communicative practices and the handling of written language using symbols, emojis, and emoticons when using mobile technologies and their different applications. Algharabali and Taqi (2018) conducted data qualitative and quantitative analysis to examine the reasons and methods of the use of emojis among college students in academic-related settings. They highlight the significant role of emojis in the discussion of college-related topics and how they can introduce a safe environment for students to vent their views and criticisms in an authorityfree setting. investigated the use of emojis in business and education, and examines how a sound instructional design approach to the development of emojis could support learning through shared visual elements in contemporary social/educational media environments.

\section{Presence of Emojis in Social Media Communication}

Cramer et al. (2016) explored the motivation for the use of emojis, their intended meaning, and functions in mobile messaging in the United States. They discussed the social and linguistic functions of emojis and denoting their complexity. According to their findings utilizing emojis can add extra emotional or social meaning to communication and boost the attractiveness of the message. Gibson (2018) proposed and highlighted the superiority of conversational analysis 
(CA) over semiotic approaches. They utilized conversational analysis (CA) instead of semiotic approaches to explore the communicative function of the 'face-covering hand' emoji and its relation to the other textual actions in face-to-face communication that affect text talk production. According to study analysis, this emoji functioned as a laughter token. There are some limitations of adopting CA in their work, the inadequate and unrealistic communicative context as they concentrating on only one emoji neglecting other emojis and their senses. Moreover, they ignore the cultural nuances between English and Chinses in their data translations and thus intermix between two different cultural assumptions and sequence properties. Cultural differences play a significant role in the use and interpretation of emojis; and some particular uses of emojis are related to the cultural background (Park et al., 2014). Finnish, Indian, and Pakistani users utilize some emojis concerning their cultural context (Sadiq et al., 2019). Pavalanathan and Eisenstein (2015) studied how emojis compete with emoticons to communicate paralinguistic content. They hypothesized that individuals who adopt emojis tend to use fewer emoticons. In their work, they utilized the causal inference approach to test their hypothesis and to know whether the use of emoticons in Twitter texts can be replaced with emojis as predefined pictographic characters. According to their results, those who adopt emojis in their texts tend to reduce their usage of emoticons rather than those who do not adopt them. Al Rashdi (2018) studied the potential forms and functions of 121 emojis as used by Omani men and women in messages exchanged on WhatsApp. The study showed that adopting emojis serve many communicative functions rather than users' emotions and females tend to use emojis more than males. Women showed an obvious tendency to use emojis with stronger emotional meanings, while men tend to use emojis with weaker emotional meanings but friendlier (Derks et al., 2008a). Herring and Dainas (2018) examined the genders (352 females, 121 males, 50 "others") differences in interpreting emojis pragmatic functions in their local discourse contexts based on the taxonomy of functions proposed by Herring and Dainas (2017). According to their study, gender did not affect appreciably the emojis' function interpretation in Facebook platform messages, and transfer from one platform to another is not necessary. Riordan (2017b) examined whether emojis of objects are also like emojis of faces to convey effect. The study stated that emojis of objects communicate positive affect, especially joy. Draw on the implication of this work, using emojis and stickers helps maintain and enhance social relations. Zhou et al. (2017) explored the proliferation of adoption and appropriation of both emojis and stickers used in mobile communication (WeChat) in Southern China. In this qualitative study and based on the cultural context, they interviewed and observed 30 participants investigate emojis use by rural and urban Chinese. Drawing on findings, emojis 
and stickers add desirable meaning to communication. Different social network platforms users tend to utilize emojis more commonly and positively on the Twitter platform than on Facebook (Hall \& Pennington, 2013).

\section{Research Methodology}

\section{Research Population and Sample}

Students from the department of English and Translation in the Faculty of Science and ArtsAlula, Madinah, Taibah University were utilized to identify and display the impact of using emojis on language in different social media applications. In this study, a convenience sample of 144 respondents has been obtained: 60 male and 83 female students both aged between 18 and $29($ mean $=23.8)$.

\section{Data Collection Instruments}

In this study, the authors utilized a survey-based questionnaire to explore the purpose of using emojis and their impact on language spelling and writing by EFL learners. The questionnaire contains two sections. Section one obtained demographic information about the students. Section two elicited information regarding the use of emojis in social media communication. The items in this questionnaire composed of different types of questions, the first question was about the frequency of which social media applications students use in their communication. The second question was about the preferred language used in communication either mother tongue or English language; and standard or dialect. The third question investigated the spelling and writing style ( emojis or language, full form of the words, or short forms and abbreviations). The fourth question was about the frequency of which social media applications students use emojis more in their communication. The fifth question was about the purpose and the context of using emojis in communication measured on a 5-point Likert scale ranging from $1=$ strongly agree to 5 strongly disagree. The final question was about the parts of speech that could be substituted by emojis among the students.

\section{Results}

The main purpose of this paper is to investigate the use and functions of emojis by EFL learners in social media and their impact on English language writing skills. This section represents the descriptive statistics of the core findings of this study derived from the methods utilized to collect and analyze the obtained data. 


\section{Emojis, Mother Tongue, and English Language use}

The first research question of this study was about the learners' preferable medium of texting in social media communication. In the first part of this question, the options given to the participants were about using Arabic standard language, Arabic dialect, and English language in social communication texting. As displayed in table one below, 53\% of male participants prefer to use their mother tongue in chatting and texting, while $3 \%$ prefer to use English. And, $47 \%$ of male participants prefer to use both their mother tongue and English language in texting. In contrast, $38 \%$ of female participants tend to utilize their mother tongue, $6 \%$ prefer to use English, and 56\% showed preference to use both their mother tongue and English in their social interaction. Table one below displays the first question responses by both male and female participants.

Table 1.

The preferable medium of texting in social media communication.

\begin{tabular}{|lcc|cc|}
\hline \multicolumn{2}{c|}{ No. } & \multicolumn{2}{c|}{ Males N= 60 } & \multicolumn{2}{c|}{$\begin{array}{l}\text { Females } \\
\text { No. }\end{array}$} \\
Item & & \multicolumn{2}{|c|}{ \%3 } \\
\hline Language choices & & & \multicolumn{3}{l|}{} \\
Mother tongue & 36 & $53 \%$ & 31 & $38 \%$ \\
English & 3 & $3 \%$ & 5 & $6 \%$ \\
Both & 21 & $47 \%$ & 47 & $56 \%$ \\
\hline Total & 60 & $100 \%$ & 83 & $100 \%$ \\
\hline Types of Texting & & & & \\
Language & 13 & $22 \%$ & 12 & $14 \%$ \\
Emojis & 2 & $2 \%$ & 11 & $13 \%$ \\
Both & 45 & $76 \%$ & 60 & $72 \%$ \\
\hline Total & 60 & $100 \%$ & 83 & $100 \%$ \\
\hline Typos & & & & \\
Yes & 13 & $22 \%$ & 11 & $27 \%$ \\
No & 14 & $24 \%$ & 12 & $18 \%$ \\
Sometimes & 23 & $54 \%$ & 60 & $55 \%$ \\
\hline Total & 60 & $100 \%$ & 83 & $100 \%$ \\
\hline
\end{tabular}

The second part of this question was about using language and emojis in social media. As noted in table one above, in male responses, $22 \%$ use language (mother tongue, dialect, and English) in their chatting, $2 \%$ of them tend to use emojis rather than language, and $76.3 \%$ utilize both language and emojis during their interaction in virtual communication. Similarly, in the case of female participants, $14 \%$ use language in texting, $13 \%$ use emojis, and $72 \%$ make use of both language and emojis when communicating in social media networks.

The third part of this question was about participants' typos handling. Table one above depicts the participants' responses, $22 \%$ of male participants are not caring about spotting 
spelling mistakes, while $24 \%$ of them are paying attention to write correctly. Simultaneously, $54 \%$ of male participants are sometimes avoid spelling mistakes in their messaging. In comparison, $27 \%$ of female participants use language in their communication without caring about their spelling or spotting writing mistakes, while, $18 \%$ use language correctly, and 55\% sometimes avoid typos.

\section{Commonly used emojis, contractions, and acronyms in social texting}

\section{Most frequent used Emojis}

The second research question was about the most frequently used emojis, contractions, and acronyms. Figure one displays the top used emojis by the participants in their interaction on various social media platforms. İt is noted that "Face with Tears of Joy " was the most used emoji by both males and females learners, while, the emoji of the " red heart $\curvearrowright$ " is used more by females rather than male learners. For the other four emojis $(\Leftrightarrow, \Theta, \Leftrightarrow$ , 3 ), it is shown that females tend to employ them more in their instant messaging.

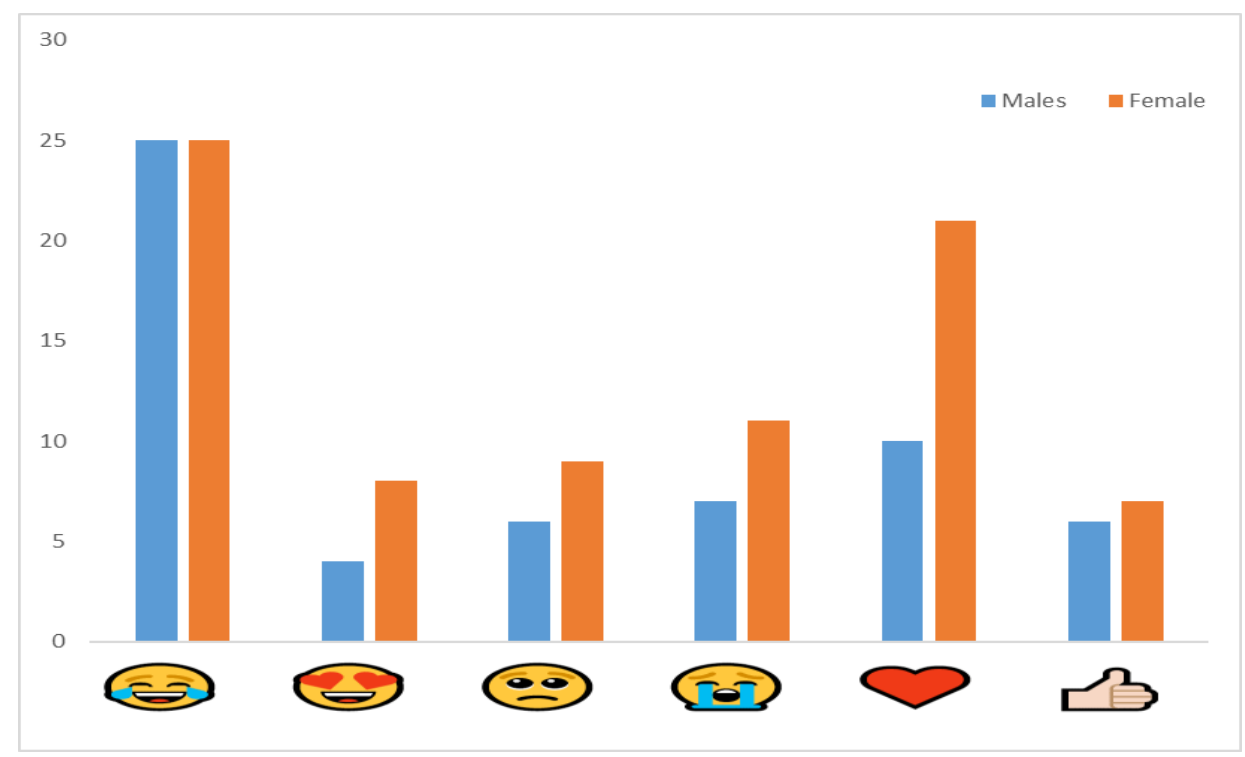

FIGURE 1.The most used emojis among male and female participants

\section{Commonly used contractions and acronyms}

Table two below displays the common abbreviations and acronyms used by learners' in their social texting. In some cases, learners replace common words, phrases, and sentences with contractions or groups of letters (acronyms) and numbers in their instant messaging because these forms help them compress their texting. İn deed, learners formed their meanings by using 
these short forms because they are more accustomed to using texting and chatting in various digital apps.

Table 2.

Common short forms used by learners in social texting

\begin{tabular}{|l|l|l|l|}
\hline Short-form & Full form & Shot Form & Full-Form \\
\hline U, u & You & Ngl & not gonna lie \\
\hline Thx & Thanks & 2morro & tomorrow \\
\hline Ty & thank you & Idk & I don't know \\
\hline r u comin & Are you coming & NP & no problem \\
\hline GM & Good Morning & LOL & laughing out loud \\
\hline rlly & really & PLS & Please \\
\hline ASAP & As soon as possible & AFAIK & As far as I know \\
\hline BTW & By the way & BRB & Br right back \\
\hline M2 & Me too & bc & Because \\
\hline TBH & To be honest & ppl & People \\
\hline hay & How are you & 4 & for \\
\hline
\end{tabular}

\section{Male and Female Frequent Used Social Media Platforms}

The third research question is about the percentage of the social media platforms use. According to the participants' responses, $92 \%$ of the male participant always use WhatsApp, the second used social media application is Snap-chat with $69 \%$ percentage, the third one is Instagram with $68 \%$ percentage, and the final used platform is Twitter with a score of $54 \%$. Most of the male Participants don't use the Facebook platform, the score of Facebook use is $5 \%$ and $39 \%$ of the participants never use Facebook Apps. Figure two below shows the scores of the male students use in different social communication networks.

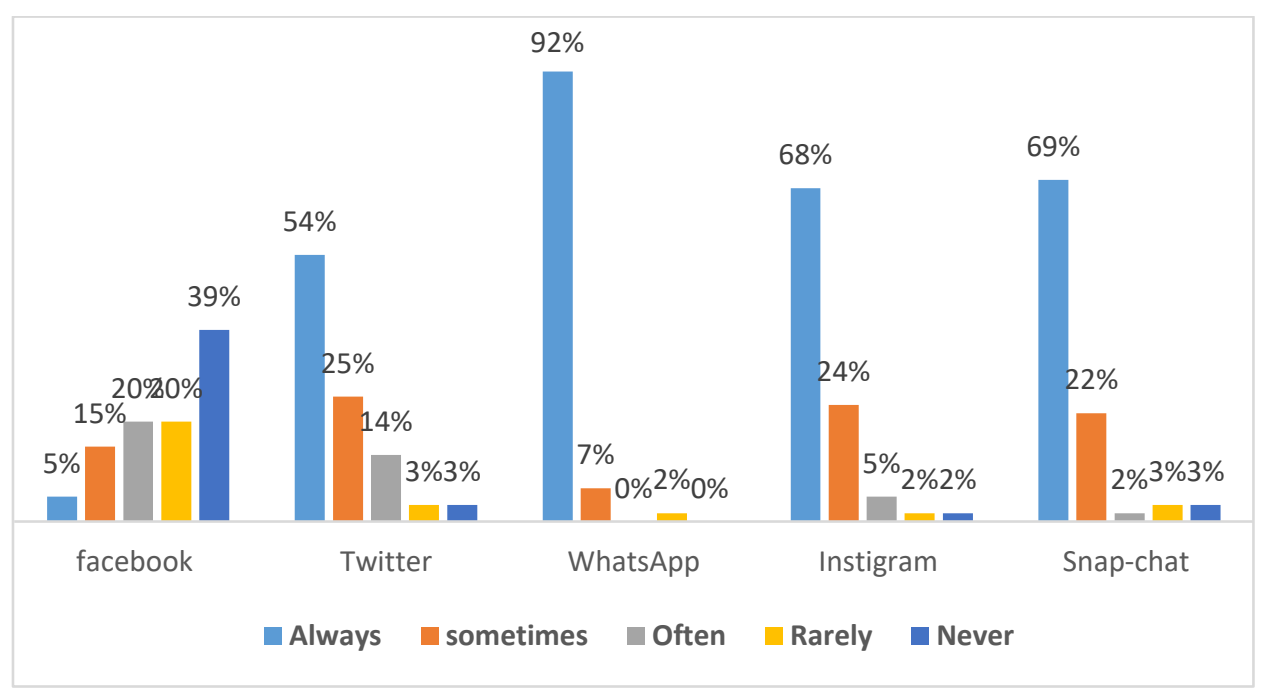

Figure 2. The Percentage of using different social networks by male participants. 
The score of the female participants' use of social media networks is displayed in figure three below. It is noticed that Snap-chat scores $78 \%$ as the highest platform for female participants who always use it, the second platform is WhatsApp with a score of $77 \%$, next is Instagram with a ratio of $70 \%$ and the final used App is Twitter with the percentage of $52 \%$. The percentage of female students who never use the Facebook app is 55\%. Figure two below displays the percentage of social media platform use.

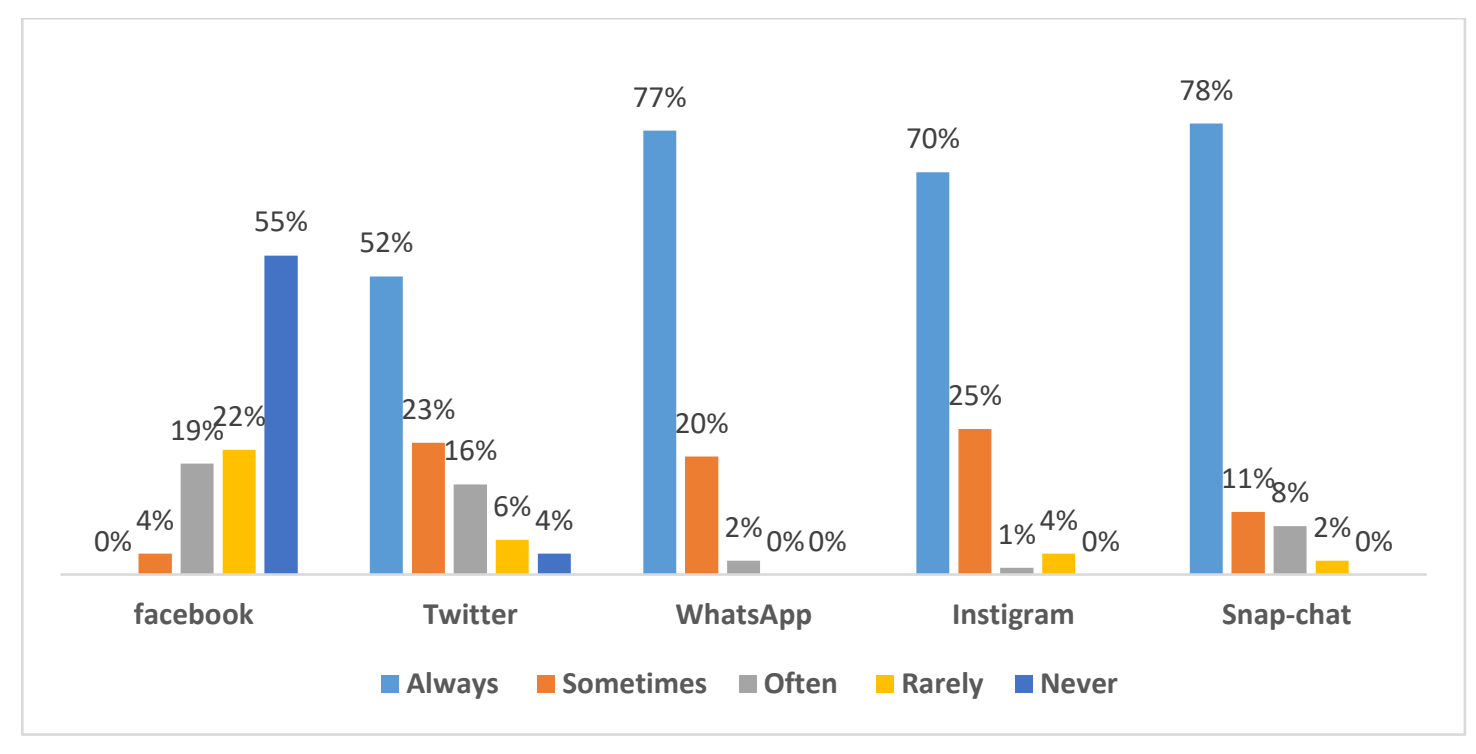

FIGURE 3.The Percentage of using different social networks by female participants.

\section{Using Emojis in Social media Platforms}

The fourth research question is about which social network applications do the male and female participants use emojis more for communication. Figure four shows the scores of using emojis in the social network. Both male and female participants use emojis more on the WhatsApp platform with a score of $81 \%$ in female cases and $71 \%$ in male cases. And the least use of emojis in social media scores in Facebook with the percentage of $9 \%$ in males' use and $6 \%$ in females. 


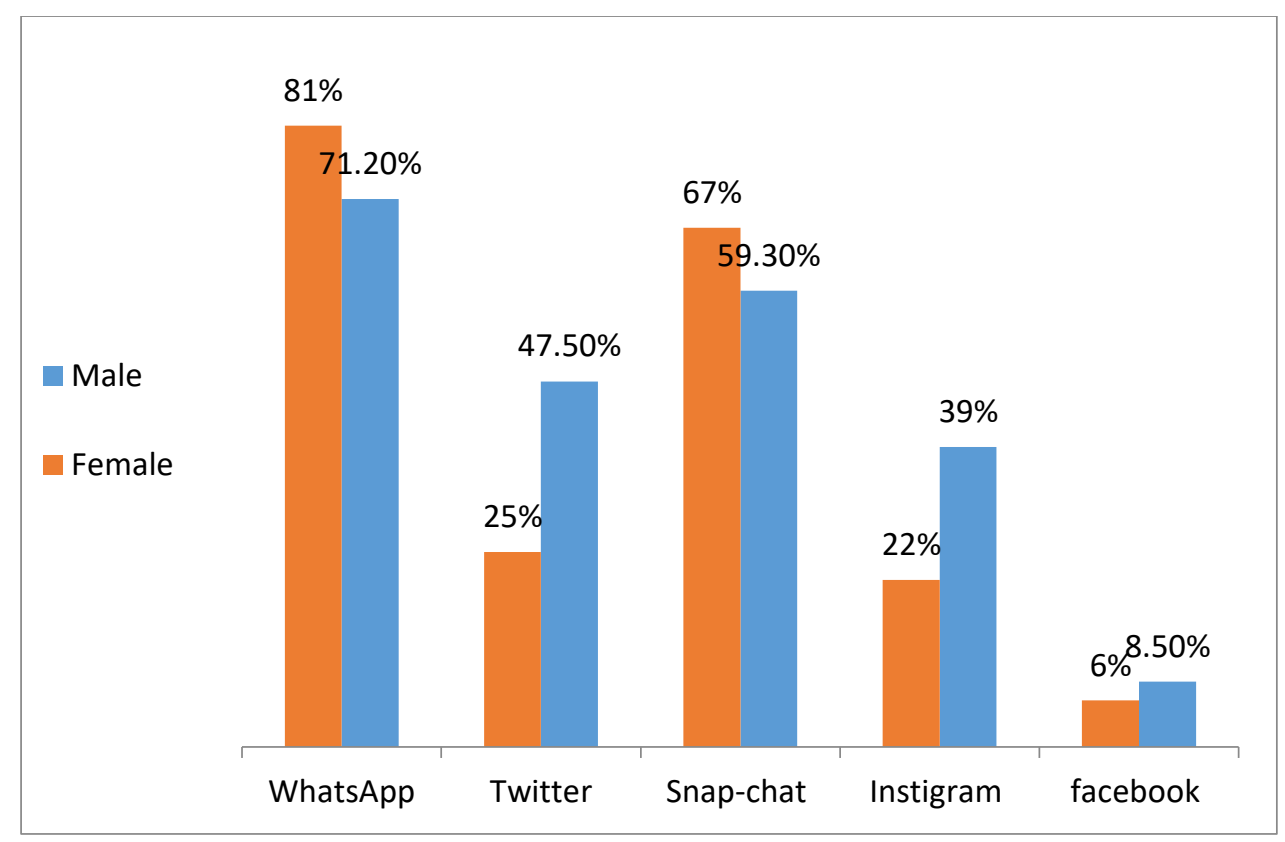

Figure 4.The Percentage of Using Emojis in a social network by male and female participants.

\section{Reasons for Using Emojis in Social Media}

The fifth research question is about the reasons for using emojis on social media platforms. Both EFL male and female learners prefer to use emojis instead of writing for several reasons that are related to social, contextual, emotional, and specific purposes. Table three displays the means' and the variances' value in male participants' responses. It is noted that there is no significant difference between these variables and the means value are the same. There is no big difference in the variance score.

Table 3.

Summary of the reasons behind using emojis by male participants.

\begin{tabular}{lccc}
\hline SUMMARY & & Males & $\mathrm{N}=60$ \\
\hline Items & Count & Mean & Variance \\
\hline $\begin{array}{l}\text { Using emojis in some social } \\
\text { context is preferable }\end{array}$ & 5 & 0.2 & 0.026487 \\
$\begin{array}{l}\text { Emojis can touch our feeling } \\
\text { more than language }\end{array}$ & 5 & 0.2 & 0.013847 \\
$\begin{array}{l}\text { Using emojis instead of } \\
\text { language in communication is } \\
\text { effective }\end{array}$ & 5 & 0.2 & 0.012267 \\
$\begin{array}{l}\text { Understanding Emojis is easier } \\
\text { during communication }\end{array}$ & 5 & 0.2 & 0.023758 \\
$\begin{array}{l}\text { Using emojis for specific } \\
\text { purposes in communication }\end{array}$ & 5 & 0.2 & 0.068141 \\
\hline
\end{tabular}


Table four below shows the analysis of variables. The $\mathrm{p}$-value of the variables is equal to one which means there is no big difference between the variables' estimation. It is much bigger than alpha. The sum of the squares between the variables is 3.33 and the sum of the errors of the square within the variables is 0.659 . The degree of freedom between the variable is 5 and the degree of freedom within the variables is 24 . The mean square of the variables is 6.66 and the means of the error is 0.027 . The F statistics score is 2.43 .

Table 4.

Analysis of Variables for the reasons of using emojis in texting by male learners.

\begin{tabular}{lrrrrrr} 
& \multicolumn{4}{c}{ Males } & \multicolumn{3}{c}{$\mathrm{N}=60$} \\
\hline & \multicolumn{1}{c}{$S S$} & $d f$ & \multicolumn{1}{c}{$M S$} & \multicolumn{1}{c}{$\begin{array}{c}\text { P- } \\
\text { Source of Variation }\end{array}$} & F crit \\
\hline Between Groups & 3.33 & 5 & 6.66 & 2.43 & 1 & 2.620654 \\
Within Groups & 0.659236 & 24 & 0.027468 & & & \\
Total & & & & & & \\
\hline
\end{tabular}

For female participants, the mean value is the same in all the variables of the reasons for using emojis in social media communication. For variances value, it is shown that the variable of emotion and effectiveness of emojis in communication scored the least value where the female participants support the use of emojis for emotional feelings and their effective use in communications as shown in table five below.

Table 5.

Summary of the reasons behind using emojis by female participants.

\begin{tabular}{lclc}
\hline SUMMARY & & Females & $\mathrm{N}=83$ \\
\hline Items & Count & Mean & Variance \\
\hline $\begin{array}{l}\text { Using emojis in some social context is } \\
\text { preferable }\end{array}$ & 5 & 0.2 & 0.042067 \\
$\begin{array}{l}\text { Emojis can touch our feeling more } \\
\text { than language }\end{array}$ & 5 & 0.2 & 0.018914 \\
$\begin{array}{l}\text { Using emojis instead of language in } \\
\text { communication is effective }\end{array}$ & 5 & 0.2 & 0.015793 \\
$\begin{array}{l}\text { Understanding Emojis is easier during } \\
\text { communication }\end{array}$ & 5 & 0.2 & 0.036769 \\
$\begin{array}{l}\text { Using emojis for specific purposes in } \\
\text { communication }\end{array}$ & 5 & 0.2 & 0.047583 \\
\hline
\end{tabular}

The sum of the squares between the variables is 2.22 and the sum of the errors of the square within the variables is 0.887 . The degree of freedom between the variables is 
5 and the degree of freedom within the variables is 24 . The mean square of the variables is 3.17 and the means of the error is 0.027 . The F statistics score is 1.14 . the $\mathrm{P}$-value is equal to one which is more than alpha .05, so, female learners are using emojis in all the above situations and they are supporting that. Table six below displays the analysis of these variables.

Table 6.

Analysis of Variables for the reasons of using emojis in texting by female learners.

Females $\mathrm{N}=83$

\begin{tabular}{lrrrrrr}
\hline \multicolumn{1}{c}{$\begin{array}{c}\text { Source of } \\
\text { Variation }\end{array}$} & \multicolumn{1}{c}{ SS } & df & MS & \multicolumn{1}{c}{ F } & P-value & F crit \\
\hline Between & & & & & & \\
Groups & 2.22 & 5 & 3.17 & 1.14 & 1 & 2.312741 \\
Within Groups & 0.887734 & 24 & 0.027742 & & & \\
& & & & & & \\
Total & 0.887734 & 29 & & & & \\
\hline
\end{tabular}

Both groups do not like to use language at these levels of communication and they prefer emojis. Emojis are more expressive than the written language with a high score in both learners' responses. All participants are supporting the touchable function of emojis where the language cannot function in the same way.

\section{Emojis effects on Language Constructs (Parts of Speech)}

The final question is about the effects of emojis on different language categories during social interaction. Learners are using emojis instead of lexical categories such as verbs, adjectives, and nouns. Figure five below depicts the score of the word-class substitution; the highest substituted utterances are verbs that have been substituted with emojis by EFL learners with the percentage of $75 \%$ in male participants and $53 \%$ in female participants. Using emojis instead of Adjectives scores 51\% in males and 56\% in females and the least substituted parts of speech are nouns with a score of $23.70 \%$ in male learners and $13 \%$ in female learners. 


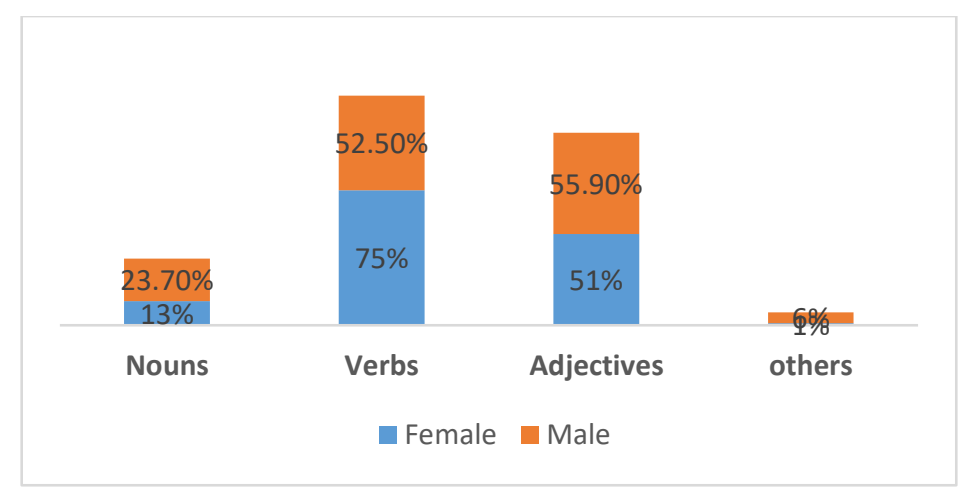

Figure 5. Using emojis instead of the Parts of speech

\section{Discussion}

This study aims to explore the impact of using emojis in social media communication on EFL learners writing skills. The study investigates the percentage of using the English language and Arabic in social media texting and chatting. The results displayed that male participants use their mother tongue more than female participants in social communication. For using English during interacting, both participants use English with a very low score. For using emojis female participants use emojis more than male participant during social texting, this study is consistent with Al Rashdi (2018) and Derks et al. (2008a) who stated that females tend to use emojis more than males and in contrast to the study of Herring and Dainas (2017) that declared gender did not affect appreciably the emojis' function interpretation in Facebook platform messages. In the case of spelling and typos, female participants pay more attention to spelling errors than male participants. The results show that the dominant social platforms used by both participants are WhatsApp, Snap-chat, Instagram, and Twitter, in contrast, Facebook is rarely used by both participants. It is noticed that both participants use emojis on most social media platforms; female participants use emojis more than male in WhatsApp and Snap-chat, whereas male participants use emojis more than female in Twitter and Instagram. For the reasons of using emojis in social media, the results reveal that emojis have rich semantics because the participants use emojis more to facilitate the communication as they convey, express views, ideas, and emotions more vividly. This view coincides with that of Lu et al. (2016) who stressed the rich semantic and cultural aspects of emojis in social media.

The p-value of these variables is equal to one which means there is no big difference between the variables' estimation. It is much bigger than alpha in the result of both male and female participants. It seems reasonable to assume that the emojis' use in social media affects the use of the language. There is a tendency among learners to use emojis instead of language. The result of the function of emojis in social media indicates that emojis can express meaning 
and feelings quickly and in better understanding as Prieto-Terrones and Sanz-Martin (2019) stressed the simplicity emojis have for rapid communication. For the linguistics forms, Learners and social network users tend to connect between the linguistic forms and their meanings naturally so that they move to use emojis more than the language in social communication. The verbs, adjectives, and nouns are more violated in social media texting and learners are moving towards more symbolic and pictographic use in social communication networks.

\section{Conclusion}

This study is set out to critically examine the ways and contexts in which EFL learners are using emojis in social media communications. The study explored the impact of emojis on language constructs and writing. The observed difference between the two groups of males and females learners is not very significant and both groups are using emojis and dialects without awareness of the importance of improving their English language writing skills through texting and chatting on social media platforms. Learners preferred to use emojis, contractions, and acronyms in their social interaction because they feel that using these mediums in their social chatting can compress and convey the message better and faster than written language. The most striking results to emerge from the data is that the standard and the second language writing are in danger because of emojis and short forms (contractions and acronyms). Likewise, there is no real attention to the threat and challenges that confront standard languages because of employing the new sorts of social communications (emojis, emoticons, slang languages, etc.) in social media texting and chatting. This work has opened up several questions that need further investigation in the impacts of these new sorts of communication on all language skills and how to orient the use of social media to improve language skills. 


\section{Reference}

Algharabali, N. A., \& Taqi, H. A. (2018). Taming the Sting: The Use of Evaluative Emojis by College Students in Kuwait. International Journal of Linguistics and Communication, 6(1), 46-60.

Al Rashdi, F. (2018). Functions of emojis in WhatsApp interaction among Omanis. Discourse, Context \& Media, 26, 117-126. DOI: 10.1016.

Bai, Q., Dan, Q., Mu, Z., \& Yang, M. (2019). A systematic review of emoji: Current research and future perspectives. Frontiers in psychology, 10, 2221.

Burholt, V., Windle, G., Gott, M., \& Morgan, D. J. (2020). Technology-mediated communication in familial relationships: Moderated-mediation models of isolation and loneliness. The Gerontologist, 60(7), 1202-1212.

Cantamutto, L. M., \& Vela Delfa, C. (2019). Emojis frecuentes en las interacciones por WhatsApp: estudio comparativo entre dos variedades de español (Argentina y España).

Cramer, H., de J. P., \& Tetreault, J. (2016, Septempe). Sender-intended functions of emojis in US messaging. In Proceedings of the 18th International Conference on Human-Computer Interaction with Mobile Devices and Services (pp. 504-509).

Danesi, M. (2016). The semiotics of emoji: The rise of visual language in the age of the internet. Bloomsbury Publishing.

Del Carmen Prieto-Terrones, P., \& Sanz-Martin, B. E. (2019). La ciberlengua empleada en WhatsApp. Unestudio de actitudes y creencias lingüísticas. Investigación y Ciencia de la Universidad Autónoma de Aguascalientes, (78), 74-84.

Derks, D., Bos, A.E., \& Von Grumbkow, J. (2007). Emoticons and social interaction on the Internet: the importance of social context. Comput. Human Behav. 23, 842- 849. https://doi.org/10.1016/j.chb.2004.11.013.

Derks, D., Bos, A. E., \& Von Grumbkow, J. (2008a). Emoticons and online message interpretation. Social Science Computer Review, 26(3), 379-388. DOI: $10.1177 / 0894439307311611$

Dunlap, J. C., Bose, D., Lowenthal, P. R., York, C. S., Atkinson, M., \& Murtagh, J. (2016). What sunshine is to flowers: A literature review on the use of emoticons to support online learning. Emotions, Technology, Design, and Learning, 163- 182.

Gibson, W., Huang, P., \& Yu, Q. (2018). Emoji and communicative action: The semiotics, sequence, and gestural actions of 'face covering hand'. Discourse Context Media 26, 91-99. DOI:10.1016/j.dcm.2018.05.005.

Giraldo Giraldo, C., Ríos Londoño, D. M., \& Cardona Cifuentes, F. A. (2018). La gramática al margen de lanorma: la escritura en WhatsApp. Lenguaje, 46(2), 311-333

Gómez del Castillo, M. T. (2017). Utilización de WhatsApp para la comunicación en titulados 
superiores. REICE. Revista Electrónica Iberoamericana sobre Calidad, Eficacia y Cambio en Educación. 15, 51-65.

Gómez Camacho, A., \& Gómez del Castillo, M. T. (2015). Escritura ortográfica y mensajes de texto enestudiantes universitarios. Perfiles educativos, 37(150), 91-104.

Herring, S. \& Dainas, A. (2017, January). "Nice picture comment!" Graphicons in Facebook comment threads. In Proceedings of the 50th Hawaii International Conference on System Sciences., 2185- 2194. IEEE.

Herring, S. C., \& Dainas, A. R. (2018, June). Receiver interpretations of emoji functions: A gender perspective. In Paper Presented at the 1st International Workshop on Emoji Understanding and Applications in Social Media (Emoji2018). Stanford, CA.

Hall, J. A., \& Pennington, N. (2013). Self-monitoring, honesty, and cue use on Facebook: the relationship with user extraversion and conscientiousness. Computers in Human Behavior. 29(4), 1556-1564. DOI: 10.1016/j.chb.2013.01.001

López Get, A. (2011). Individuación y lenguaje no verbal en la CMC.

Lu, X., Ai, W., Liu, X., Li, Q., Wang, N., Huang, G., \& Mei, Q. (2016, September). Learning from the ubiquitous language: an empirical analysis of emoji usage of smartphone users. In Proceedings of the 2016 ACM International Joint Conference on Pervasive and Ubiquitous Computing (pp. 770-780).

Mahyoob, M. (2020).Challenges of e-Learning during the COVID-19 Pandemic Experienced by EFL Learners. Arab World English Journal, 11 (4) 351-362.

Martínez-Lirola, M. (2012). Aproximación a la interacción virtual: el caso de la red social Badoo. Palabra Clave, 15(1), 107-127.

Pavalanathan, U., \& Eisenstein, J. (2015). Emoticons vs. emojis on Twitter: A causal inference approach. arXiv preprint arXiv:1510.08480.

Park, J., Baek, Y. M., and Cha, M. (2014). Cross-cultural comparison of nonverbal cues in emoticons on Twitter: evidence from big data analysis. J. Commun. 64, 333-354. DOI: $10.1111 /$ jcom. 12086

Riordan, M. A. (2017b). Emojis as tools for emotion work: communicating affect in text messages. $J$. Lang. Soc. Psychol. 36, 549-567. DOI: 10.1177/0261927X17704238.

Rodríguez Morales, Z., \& Rodríguez Salazar, T. (2016). Los jóvenes, la comunicaciónafectiva y las tecnologías: entre la ritualización de la expresión y la regulación emocional. Intersticios sociales, (11), 0-0.

Sadiq, M. (2019, January). Learning Pakistani culture through the namaz emoji. In 2019 2nd International Conference on Computing, Mathematics and Engineering Technologies 
(iCoMET) (pp. 1-8). IEEE. DOI: 10.1109/ICOMET.2019.8673479.

Thompson, D., \& Filik, R. (2016). Sarcasm in written communication: Emoticons are efficient markers of intention. Journal of Computer-Mediated Communication, 21(2), 105-120. https://doi.org/10.1016/j.chb.2004.11.013

Veytia-Bucheli, M. G., Gómez-Galán, J., \& Vergara, D. (2020). Presence of new forms of intercultural communication in Higher Education: Emojis and social interactions through WhatsApp among graduate students. Education Sciences, 10(11), 295.

Zhou, R., Hentschel, J., \& Kumar, N. (2017, May). Goodbye text, hello emoji: mobile communication onwechat in China. In Proceedings of the 2017 CHI Conference on Human Factors in Computing Systems. pp. 748-759).DOI: 0.1145/3025453 